\title{
Possible implications of maternal HIV infection for increasing measles susceptibility in young infants
}

\author{
Sabelle Jallow ${ }^{1,2} \&$ Shabir A Mahdi*,1,3 \\ ${ }^{1}$ Medical Research Council: Respiratory \& Meningeal Pathogens Research Unit, Faculty of Health Sciences, University of \\ Witwatersrand, Johannesburg, South Africa \\ ${ }^{2}$ Centre for Vaccines \& Immunology, National Institute for Communicable Diseases of the National Health Laboratory Service, \\ Johannesburg, South Africa \\ ${ }^{3}$ Department of Science \& Technology/National Research Foundation: Vaccine Preventable Diseases, Faculty of Health Sciences, \\ University of the Witwatersrand, South Africa \\ * Author for correspondence: Tel.: 002711983 4283; madhis@rmpru.co.za
}

\begin{abstract}
“'Recent outbreaks in LMICs, such as South Africa, observed a demographic shift in measles cases; in addition to high percentage of cases occurring in very young infants ( $24 \%$ of 18,431 laboratory confirmed cases in infants less than 9 months age) [6]; adults in the age group of high HIV prevalence were also affected ( $20 \%$ in adults aged $15-39$ years) [7]."
\end{abstract}

First draft submitted: 5 February 2018; Accepted for publication: 5 March 2018; Published online: 21 May 2018

Keywords: 6 months of age $\bullet$ dosing schedule $\bullet$ HIV $\bullet$ measles $\bullet$ naturally acquired immunity $\bullet$ transplacental antibody transfer $\bullet$ vaccine $\bullet$ vaccine dose $\bullet$ vaccine-induced immunity

Measles remains a leading cause of vaccine preventable morbidity and mortality in children [1]. Despite recent progress in improving measles vaccine coverage in children, particularly in low and middle-income countries (LMICs) [1], in 2015 measles incidence was estimated at 9.7 million cases and 134,200 (95\% CI of 74,400$353,600)$ deaths [2]. Although measles is targeted for eradication, this remains elusive, partly because of the highly contagious nature of measles; requiring at least $95 \%$ vaccine coverage to interrupt transmission [3]. Instead, there has been a reduction in measles vaccine coverage recently, which has resulted in outbreaks, even in high-income countries, where measles cases have been uncommon in the past decade [4,5]. Recent outbreaks in LMICs, such as South Africa, observed a demographic shift in measles cases; in addition to high percentage of cases occurring in very young infants ( $24 \%$ of 18,431 laboratory confirmed cases in infants less than 9 months age) [6]; adults in the age group of high HIV prevalence were also affected (20\% in adults aged $15-39$ years) [7]. This demographic shift might relate to waning of measles immunity, including among women in the reproductive age-group, who have primarily derived immunity through vaccination rather than wild-type virus exposure, resulting in lower antibody titers transferred to their newborns $[8,9]$.

Increased maternal age has been showed to be correlated with higher transplacental transfer of measles antibodies [9] due to older women being more likely to have acquired immunity to measles through wild-type virus exposure $[8,9]$. Furthermore, vaccine-induced immunity wanes faster than naturally acquired immunity [8], resulting in lower concentrations of transplacental measles antibody acquisition in newborns of women who derive immunity through vaccination. Reportedly, $99 \%$ of newborns from women with vaccine-acquired immunity have undetectable measles antibodies by 6 months of age [8], resulting in increased susceptibility at a younger age in infants. The role of maternal-derived antibodies in providing protection against infectious diseases in young infants is well established. Maternal $\operatorname{IgG}$ is actively transported across the placenta mainly during the third trimester and can be present at levels as high or even higher than in the mother [10]. Transplacental antibody transfer is, however, influenced by several factors, including maternal antigen-specific IgG levels, IgG subclass, gestational age and placental integrity, which could be affected by HIV and malaria [10]. During HIV infection, formation of immune complexes may cause physical blockage of the FcRn receptors and impairment of IgG, including developmental 
and avidity functions [11], which may lead to the inability of $\operatorname{IgG}$ to bind FcRn receptors and consequently, reduce transfer of IgG across the placenta. Moreover, placental inflammation during HIV has also been associated with reduced placental integrity [12], which in turn lowers transplacental transfer efficiency.

The HIV epidemic may have enhanced measles transmission [13]. Measles disease in HIV-infected children is characterized with unusual and severe clinical manifestations, including a high frequency of pneumonitis and measles-associated neurological disorders [13]. Furthermore, in the USA, case fatality rate of measles was higher in HIV-infected $(37 \%)$ individuals when compared with the general population $(<1 \%)$ [13]. HIV-infected children also have lower levels of measles antibodies that appear to decline faster than in healthy HIV-uninfected children [13]. Prospective trials in the USA to a single dose of standard titer measles vaccine in HIV-infected children suggested an impaired immune response to the vaccine with a proportion of vaccinated children remaining susceptible to measles disease [13]. A study in Kenya, where only 33\% of antiretroviral-naive HIV-infected children had seroprotective measles antibodies $2-5$ years after vaccination in their first year of life, indicated waning of immunity and possible impairment of maintained B-cell memory responses [14]. HIV-infected children have also been shown to have prolonged viral shedding, which may lead to increased transmission [13]. Taken together, HIV infection may have increased the incidence of measles, including outbreaks.

In sub-Saharan Africa, this increased susceptibility of young infants to measles infection and consequently, more severe disease, might be compounded by the high prevalence of infants born to HIV-infected women, in whom immunosuppression results in greater waning of measles and other vaccine preventable disease antibodies [15]. The WHO recommended a measles vaccine dosing schedule for LMICs which involves delaying vaccination until at least 9 months of age with a booster dose at the second year of life [1]. This was largely premised on the interference of maternal-derived measles IgG antibodies on the immunogenicity of the vaccine at a younger age [16] and compounded by the innately lower immunogenicity of measles vaccine in young infants due to their immature immune systems [16]. The success of mother-to-child HIV prevention programs has reduced the risk of HIV infection in infants born to HIV-infected women to $<1 \%$ [17]. The prevalence of HIV infection among pregnant women in many sub-Saharan African countries has, however, remained unchanged in the past decade resulting in high numbers of HIV-exposed uninfected infants, who are at an increased risk of infection-related morbidity and mortality during infancy, possibly due to aberrant immune systems [17] and also reduced transplacental acquisition of protective maternal IgG against vaccine-preventable diseases [9]. In South Africa, where maternal HIV prevalence is $30 \%$ [7], prevaccination at about 20 weeks of age resulted in only $28.8 \%$ and $27.7 \%$ of infants born to HIVuninfected and HIV-infected women, respectively, having detectable measles IgG; with an even lower proportion (16.7\% and $13.8 \%$, respectively) having sero-protective measles antibody titers [18]. Furthermore, at 10-month postvaccination, $94.3 \%$ and $79.6 \%$ of HIV-unexposed uninfected (HUU) and HIV-exposed uninfected (HEU) infants, respectively, had sero-protective measles antibody titers [18]. Similarly, in Rwanda, only $10 \%$ of HEU compared with $25 \%$ of HUU infants had detectable measles antibodies prevaccination at 6 months of age [19]. Until recently (2017), WHO only recommended measles vaccination at 6 months of age and booster doses at 9 months and 12-15 months in HIV-infected children [1]. However, with evidence of lower measles antibody levels in HEU children [9], and therefore earlier susceptibility to measles disease, the current recommendation of vaccination at 6 months of age includes both HIV-infected and HIV-exposed uninfected children [1]. This schedule has, however, not yet been widely adopted, including in settings of high HIV prevalence. Furthermore, based on the waning immunity seen even in HIV-uninfected women; who derived immunity from vaccination - this dosing strategy might also need to be expanded to HIV-unexposed infants, particularly in the absence of high ( $>98 \%)$ community coverage. Consequently, although the measles vaccine has been available since the 1960 s, studies informing current dosing schedules might need to be reconsidered in context of the changes in measles immunity among women of child-bearing age, especially in the areas of high HIV prevalence.

The changing sero-epidemiological aspects of measles dictates that we explore different dosing schedules; including having the first measles vaccine dose at 6 months of age; even though this might be offset by the innately lower immunogenicity of the vaccine. Protection from measles after natural infection and vaccination involves both humoral and cellular-immune pathways; so that even among infants with a poor antibody response, there could be benefit of priming $\mathrm{B}$ and $\mathrm{T}$ cells in order to provide better responses upon re-exposure [20]. Furthermore, this earlier vaccine approach may require new measles vaccines that are not only more immunogenic, but also less likely to be affected by 'blunting' - the reduction in vaccine efficacy due to the presence of low levels of maternal-derived antibodies. Also, consideration should be given toward providing a booster dose to nonpregnant women of reproductive age, especially perhaps in HIV-infected women (and men) in LMICs. This will provide protection to both 
mothers and infants in cases of outbreaks, as well as increase the transplacental-transferred measles antibodies to the infant, increasing the duration of passive protection from maternally derived antibodies. Furthermore, the optimal dosing schedule of both the first vaccination at 6 months of age and the frequency/timing of future booster doses requires further examination, especially in settings where maternal HIV prevalence is high.

\section{Financial \& competing interests disclosure}

The authors have no relevant affiliations or financial involvement with any organization or entity with a financial interest in or financial conflict with the subject matter or materials discussed in the manuscript. This includes employment, consultancies, honoraria, stock ownership or options, expert testimony, grants or patents received or pending, or royalties.

No writing assistance was utilized in the production of this manuscript.

\section{References}

1 Measles vaccines: WHO position paper - April 2017. Wkly Epidemiol. Rec. 92(17), 205-227 (2017).

2 Patel MK, Gacic-Dobo M, Strebel PM et al. progress toward regional measles elimination - worldwide, 2000-2015. MMWR Morb. Mortal Wkly Rep. 65(44), 1228-1233 (2016).

3 Adamo G, Sturabotti G, D’Andrea E et al. The end of measles and congenital rubella: an achievable dream? Ann. Ig. 29(1), 1-26 (2017).

4 European Centre for Disease Prevention and Control. Epidemiological update: measles - monitoring European outbreaks, 7 July 2017. www.ecdc.europa.eu/en/news-events/epidemiological-update-measles-monitoring-european-outbreaks-7-july-2017

5 Gans HA, Maldonado YA. Loss of passively acquired maternal antibodies in highly vaccinated populations: an emerging need to define the ontogeny of infant immune responses. J. Infect. Dis. 208(1), 1-3 (2013).

6 Ntshoe GM, McAnerney JM, Archer BN et al. Measles outbreak in South Africa: epidemiology of laboratory-confirmed measles cases and assessment of intervention, 2009-2011. PLoS ONE 8(2), e55682 (2013).

7 Barron P, Pillay Y, Doherty T et al. Eliminating mother-to-child HIV transmission in South Africa. Bull. World Health Organ. 91(1), 70-74 (2013).

8 Leuridan E, Hens N, Hutse V, Ieven M, Aerts M, Van Damme P. Early waning of maternal measles antibodies in era of measles elimination: a longitudinal study. BMJ 340, c1626 (2010).

9 Jallow S, Cutland CL, Masbou AK, Adrian P, Madhi SA. Maternal HIV infection associated with reduced transplacental transfer of measles antibodies and increased susceptibility to disease. J. Clin. Virol. 94 50-56 (2017).

10 Palmeira P, Quinello C, Silveira-Lessa AL, Zago CA, Carneiro-Sampaio M. IgG placental transfer in healthy and pathological pregnancies. Clin. Dev. Immunol. 2012, 985646 (2012).

11 Nair N, Moss WJ, Scott S et al. HIV-1 infection in Zambian children impairs the development and avidity maturation of measles virus-specific immunoglobulin $\mathrm{G}$ after vaccination and infection. J. Infect. Dis. 200(7), 1031-1038 (2009).

12 St Louis ME, Kamenga M, Brown C et al. Risk for perinatal HIV-1 transmission according to maternal immunologic, virologic, and placental factors. JAMA 269(22), 2853-2859 (1993).

13 Moss WJ, Cutts F, Griffin DE. Implications of the human immunodeficiency virus epidemic for control and eradication of measles. Clin. Infect. Dis. 29(1), 106-112 (1999).

14 Farquhar C, Wamalwa D, Selig S et al. Immune responses to measles and tetanus vaccines among Kenyan human immunodeficiency virus type $1(\mathrm{HIV}-1)$-infected children pre and post highly active antiretroviral therapy and revaccination. Pediatr. Infect. Dis. J. 28(4), 295-299 (2009).

15 Jones CE, Naidoo S, De Beer C, Esser M, Kampmann B, Hesseling AC. Maternal HIV infection and antibody responses against vaccine-preventable diseases in uninfected infants. JAMA 305(6), 576-584 (2011).

16 Caceres VM, Strebel PM, Sutter RW. Factors determining prevalence of maternal antibody to measles virus throughout infancy: a review. Clin. Infect. Dis. 31(1), 110-119 (2000).

17 Abu-Raya B, Kollmann TR, Marchant A, MacGillivray DM. the immune system of HIV-exposed uninfected infants. Front. Immunol. 7383 (2016).

18 Simani OE, Adrian PV, Violari A et al. Effect of in-utero HIV exposure and antiretroviral treatment strategies on measles susceptibility and immunogenicity of measles vaccine. AIDS 27(10), 1583-1591 (2013).

19 Lepage P, Dabis F, Msellati P et al. Safety and immunogenicity of high-dose Edmonston-Zagreb measles vaccine in children with HIV-1 infection. A cohort study in Kigali, Rwanda. Am. J. Dis. Child. 146(5), 550-555 (1992).

20 Bertley FM, Ibrahim SA, Libman M, Ward BJ. Measles vaccination in the presence of maternal antibodies primes for a balanced humoral and cellular response to revaccination. Vaccine 23(4), 444-449 (2004). 
